\title{
Grand challenge for ion channels: an underexploited resource for therapeutics
}

\section{Diana Conte Camerino* and Jean-François Desaphy}

Section of Pharmacology, Department of Pharmacobiology, Faculty of Pharmacy, University of Bari "Aldo Moro," Bari, Italy

${ }^{*}$ Correspondence: conte@farmbiol.uniba.it

In the last decade, only a few new ion channel drugs have reached the market according to the Food and Drug Administration. These include nateglinide, a non-sulfonylurea blocker of KATP channel used in type II diabetes; ziconotide, a N-type calcium channel blocker against severe chronic pain; pregabalin, a calcium channel $\alpha 2 \delta$ subunit ligand indicated for neuropathic pain; ranolazine, a blocker of late sodium current for chronic angina pectoris; and lubiprostone, a ClC-2 chloride channel activator for chronic idiopathic constipation. In the meantime, the sodium channel blocker mexiletine, indicated as a class IIb antiarrhythmic drug but used off-label in many disorders of membrane excitability, was withdrawn from the market except in Japan. While a clinical trial was launched last year to confirm the effectiveness of mexiletine against muscle stiffness in nondystrophic myotonia, myotonic patients, and their physicians have now to consider shifting to other drugs and defining new therapy rules.

Nonetheless it is widely acknowledged that research activity of the pharmaceutical community in ion channel field has been tremendously increasing in recent years (Conte Camerino et al., 2007). Such activity continues to investigate sectors traditionally covered by ion channel drugs, such as cardiovascular diseases, epilepsies, neuropathic pain, and diabetes. For instance, new calcium channel blockers, which constitute one of the major classes of therapeutic agents, have been identified by pharmacological and radiochemical techniques and largely studied by patch-clamp. Thus this research field is not abandoned and novel compounds are currently considered (Godfraind, 2005, 2006). Now it also regards new sectors with great potential such as cancer, inflammation, immunomodulation, kidney diseases, and pathogen infection. In addition, ion channels are straightforward druggable targets for a number of rare diseases, including the ion channelopathies.
Ion channelopathies are monogenic hereditary diseases due to mutations in genes encoding ion channel subunits (Conte Camerino et al., 2008). The term was coined about 20 years ago to define the skeletal muscle disorders due to mutations in sodium, chloride, and calcium genes with symptoms ranging from loss of excitability to over-excitability. Since then many other genetic diseases affecting the whole range of tissues have been linked to ion channel gene defects. The discovery of ion channelopathies may be considered as a revolution for the study of ion channels, the third one after the pioneering work of Nobel prize-winning Hodgkin and Huxley in the 1950s, who have defined the basis for selective ion currents across the plasma membrane, and the invention of the patchclamp technique by Neher and Sakmann in the 1970s, Nobel prize in 1991, which allows to record any kind of ion channel activity in almost all cell types (Hodgkin and Huxley, 1952; Hamill et al., 1981). An other recent outstanding advancement in ion channel research was the 3D resolution of channel structure by X-ray crystallography (MacKinnon, 2004).

The discovery of channelopathies coincides with the combination of electrophysiological and molecular genetic techniques. The possibility to study the dysfunction of a mutated channel in an heterologous system of expression and to correlate it with the clinical phenotype have tremendously increased our knowledge about the role of ion channel in physiology and pathology. Importantly the ion channelopathies can also serve as paradigms to understand the more complex and frequent multifactorial diseases. At the same time, the use of knockdown and knock-out animal experiments have also significantly contributed to the understanding of ion channel contribution to diseases. Altogether these progresses have underscored the therapeutic potential of ion channel modulators. Drugs acting on ion channels were already in use before the channelopathies were known, but most of these drugs were used empirically and were found to act on ion channels afterward. Now our knowledge about their role in diseases have validated ion channels as very promising druggable targets.

What are the difficulties encountered in ion channel drug discovery? A major problem of ion channel drugs regards the side effects. An ion channel type is often expressed in many cell types, and it is important to target the ill tissue without altering the normal function of the others. Let's take the example of voltage-gated sodium channels, which are expressed in many excitable tissues including the heart and the central nervous system. The use of sodium channel blockers to counteract hyper-excitability in peripheral tissues, such as the skeletal muscle in myotonia or the nociceptive neuron in neuropathic pain, can be significantly limited by the side action on cardiac or CNS sodium channels. Thus the International Association for the Study of Pain recommends orally available sodium channel blockers as third-line treatment in neuropathic pain, mainly because of their potential side effects (Dworkin et al., 2007). Up to now, to limit these unwanted effects, a peculiarity of sodium channel blockers was exploited, that is the use-dependence - the higher the frequency of action potential firing, the greater is the block of sodium channels - allowing a rather specific action of drugs in hyperexcited cells. A more recent successful therapy based on a channel gating-dependent mechanism of action is that of ranolazine in angina pectoris, which appears to inhibit quite specifically the guilty late sodium current that is increased in the ischemic myocyte (Chaitman, 2006). An other manner to limit the side effects is to restrain body exposure when it is possible, such as with topical lidocaine for post-herpetic pain or intrathecal ziconotide for severe chronic pain. On an other hand, the search for drugs specific to channel subtypes has 
proven challenging. For instance, nine genes in the human genome are known to encode voltage-gated sodium channel subtypes with high sequence homology, which are expressed in a tissue-selective manner. No specific drug for the skeletal muscle hNav1.4 sodium channel is available yet, and drugs relatively selective for hNav1.7 and hNav1.8 channels, which are mainly expressed in peripheral nociceptive neurons, have been described only very recently (Jarvis et al., 2007; Williams et al., 2007).

Drug screening on ion channels has been a slow process for a long time, due to the lack of high throughput techniques. The gold standard for studying ion channel function and pharmacology is the patch-clamp technique (Hamill et al., 1981). Although the amazing signal and time resolutions of this technique allow an in-depth description of intimate drug-channel interaction, it remains a laborious and time consuming experiment requiring skilled operators, which limits its application as a screening platform. In the last decade, high throughput automated electrophysiological platforms have been developed, which are more suited for large drug screenings such as those performed in the modern pharmaceutical industry (Castle et al., 2009). This is expected to accelerate the individuation and lead optimization of drug candidates. Nevertheless, because of the complex interaction of drugs with channel gating, it is likely that the patch-clamp technique will remain irreplaceable for many years in many circumstances. This later consideration is especially true for searching drug candidates in ion channelopathies, because the mutations may modify drug-channel interaction either directly or secondarily to alteration of gating. More drastically, voltage-sensor mutations of hNav1.4 sodium channels responsible for periodic paralysis have been shown to create a new pathway for cations parallel to the sodium ion conducting pore of the channel, which implies searching drugs acting radically differently on mutant compared to wild-type channels (Sokolov et al., 2010). Moreover, other technologies would be also needed to screen ion channels for pharmacological chaperones able to improve ion channel surface membrane expression, which may be impaired by pathogenic mutations (Amaral, 2006).

In addition to pharmacotherapy, ion channels have been often neglected as targets for toxicants or as undesired targets for drugs. Now, preclinical testing is required by the FDA and other agencies for registration of pharmaceuticals, which consists in assessing the risk potential of the test substance to block cardiac hERG potassium channels and delay ventricular repolarization. Many pharma/biotech(s) also routinely test their drug candidates on cardiac hNav1.5 sodium channels before to go ahead in development. This may allow to discard potential cardiotoxic compounds early during drug development. The progress of high throughput systems to investigate ion channel pharmacology will certainly allow to increase this kind of toxicological assays.

In conclusion, the human genome project has identified more than 400 genes encoding ion channel subunits, and many have been already shown to play a critical role in diseases, either directly or indirectly. It is likely that this knowledge will further increase by taking advantage of the most modern methodologies, such as the omics technologies. In the next future, the grand challenge of ion channel research will consist in finding more specific drugs able to block selectively ion channel subtypes or ion channel mutants to allow the development of new and safer pharmacotherapy and concretize ion channel pharmacogenetics from the bench side to the clinics.

\section{REFERENCES}

Amaral, M. D. (2006). Therapy through chaperones: sense or antisense? Cystic fibrosis as a model disease. J. Inherit. Metab. Dis. 29, 477-487.

Castle, N., Printzenhoff, D., Zellmer, S., Antonio, B., Wickenden, A., and Silvia, C. (2009). Sodium channel inhibitor drug discovery using automated high throughput electrophysiology platforms. Comb. Chem. High Throughput Screen. 12, 107-122.

Chaitman, B. R. (2006). Ranolazine for the treatment of chronic angina and potential use in other cardiovascular conditions. Circulation 113, 2462-2472.

Conte Camerino, D., Desaphy, J.-F., Tricarico, D., Pierno, S., and Liantonio, A. (2008). Therapeutic approaches to ion channel diseases. Adv. Genet. 64, 81-145.

Conte Camerino, D., Tricarico, D., and Desaphy, J.-F. (2007). Ion channel pharmacology. Neurother. 4, 184-198.
Dworkin, R. H., O’Connor, A. B, Backonja, M., Farrar, J. T., Finnerup, N. B., Jensen, T. S., Kalso, E. A., Loeser, J. D., Miaskowski, C., Nurmikko, J. T. J., Portenoy, R. K., Rice, A. S. C., Stacey, B. R., Treede, R.-D., Turk, D. C., and Wallace, M. S. (2007). Pharmacologic management of neuropathic pain: evidence-based recommendations. Pain 132, 237-251.

Godfraind, T. (2005). Antioxidant effects and the therapeutic mode of action of calcium channel blockers in hypertension and atherosclerosis. Phil. Trans. $R$. Soc. B 360, 2259-2272.

Godfraind, T. (2006). Calcium-channel modulators for cardiovascular disease. Expert Opin. Emerg. Drugs 11, 49-73.

Hamill, O. P., Marty, A., Neher, E., Sakmann, B., and Sigworth, F. J. (1981). Improved patch-clamp techniques for high-resolution current recording from cells and cell-free membranes patches. Pflugers Arch. 391, 85-100.

Hodgkin, A. L., and Huxley, A. F. (1952). A quantitative description of membrane current and its application to conduction and excitation in nerve. J. Physiol. 117, 500-544.

Jarvis, M. F., Honore, P., Shieh, C. C., Chapman, M., Joshi, S., Zhang, X. F., Kort, M., Carroll, W., Marron, B., Atkinson, R., Thomas, J., Liu, D., Krambis, M., Liu, Y., McGaraughty, S., Chu, K., Roeloffs, R., Zhong, C., Mikusa, J. P., Hernandez, G., Gauvin, D., Wade, C., Zhu, C., Pai, M., Scanio, M., Shi, L., Drizin, I., Gregg, R., Matulenko, M., Hakeen, A., Gross, M., Johnson, M., Marsh, K., Wagoner, K., Sullivan, J. P., Faltynek, C. R., and Krafte, D. (2007). A-803467, a potent and selective Nav1.8 sodium channel blocker, attenuates neuropathic and inflammatory pain in the rat. Proc. Natl. Acad. Sci. U.S.A. 104, 8520-8525.

MacKinnon, R. (2004). Nobel lecture. Potassium channels and the atomic basis of selective ion conduction. Biosci. Rep. 24, 75-100.

Sokolov, S., Scheuer, T., and Catteral, W. A. (2010). Ion permeation and block of the gating pore in the voltage sensor of Nav1.4 channels with hypokalemic periodic paralysis mutation. J. Gen. Physiol. 136, 225-236.

Williams, B. S., Felix, J. P., Priest, B. T., Brochu, R. U., Dai, K., Hoyt, S. B., London, C., Tang, Y. S., Duffy, J. L., Parsons, W. H., Kaczorowski, G. J., and Garcia, M. (2007). Characterization of a new class of potent inhibitors of the voltage-gated sodium channel Nav1.7. Biochemistry 46, 14693-14703.

Received:06August 2010; accepted:06 August 2010; published online: 20 August 2010.

Citation: Camerino DC and Desaphy J-F. (2010) Grand challenge for ion channels: an underexploited resource for therapeutics. Front. Pharmacol. 1:113. doi: 10.3389/ fphar.2010.00113

This article was submitted to Frontiers in Ion Channel and Channelopathies, a specialty of Frontiers in Pharmacology.

Copyright (c) 2010 Camerino and Desaphy. This is an open-access article subject to an exclusive license agreement between the authors and the Frontiers Research Foundation, which permits unrestricted use, distribution, and reproduction in any medium, provided the original authors and source are credited. 